\title{
Adverse Drug Reactions and Avalanches: Life at the Edge of Chaos
}

\author{
Daniel A. C. Frattarelli, MD
}

\begin{abstract}
Although many reports have described the incidence of adverse drug reactions, none have explained their variable severity or why they happen. Because human physiology shares many of the features of other complex adaptive systems, reactions to drug therapy were examined mathematically for specific patterns to show (1) that the severity of adverse drug reactions follows a distribution seen in other complex adaptive systems, called a power law distribution, and (2) that preventable reactions occurred for reasons fundamentally different from those that underlie the nonpreventable reactions. Two reports detailing adverse drug reaction incidence and severity were evaluated: a meta-analysis of prospective adverse drug reaction studies and a prospective cohort study. Incidence of drug reaction was plotted as a function of severity and fit to an equation. The incidences of overall and nonpreventable drug reaction, plotted as a function of severity, followed a similar power law distribution regardless of
\end{abstract}

sample size or the nature of the population or drugs studied. An exception to this was the preventable reactions, which were described by a different type of equation. Response to pharmacotherapy exhibits many properties of systems with self-organized criticality. An exception to this is the preventable reactions, which seem to be fundamentally different from the nonpreventable ones. These observations suggest that the presence and the distribution of severity of reaction to pharmacotherapy is a consequence of our adaptation as biological systems, and although adverse reactions can be made less frequent, a certain percentage will not be preventable.

Keywords: Adverse drug reactions; power laws; complex systems; systems biology

Journal of Clinical Pharmacology, 2005;45:866-871 @2005 the American College of Clinical Pharmacology

A dverse drug reactions (ADRs) are a significant concern, with considerable attendant morbidity and mortality. ${ }^{1-5}$ Although several reports have described the incidence of these reactions, there has not been any satisfactory explanation offered as to the distribution of the variable severity of these reactions (eg, why are milder reactions more frequent than fatal ones?) or even to explain why they happen at all.

Many complex systems have been studied in terms of their response to perturbation. These systems have many features that are similar to those that describe human physiology: they are adaptable, maintain a state of dynamic nonequilibrium, consist of many diverse agents acting together in a network, and exhibit a pattern of behavior that emerges from and is greater than the sum of its parts. Well-studied examples of this phe-

From Wayne State University School of Medicine, Division of Clinical Pharmacology and Toxicology, Children's Hospital of Michigan, Detroit. Submitted for publication December 15, 2004; revised version accepted April 25, 2005. Address for reprints: Daniel A. C. Frattarelli, MD, Wayne State University School of Medicine, Division of Clinical Pharmacology and Toxicology, Children's Hospital of Michigan, 3901 Beaubien, Detroit, Ml 48201. DOI: $10.1177 / 0091270005278056$

nomenon exist in physics and in the political and economic sciences. $^{6}$

Because human physiology shares many of the same features of other systems that have benefited from such analysis, the patterns of reaction to drug therapy were examined. Response to pharmacotherapy was chosen because it is the closest approximation of the sort of systematic perturbation of a complex system that we have in regular medical practice. As the methods of identifying patterns associated with these systems work best with larger numbers of data points, 2 large reports detailing the incidence and severity of ADR were examined.

Two hypotheses were tested. The first was that the severity of ADR follows a mathematical distribution seen in other complex adaptive systems, called a power law distribution (so named because the equation that describes these processes shows the dependant variable to change as a function of the independent variable raised to a fixed power, such as $\left.y=x^{2}\right){ }^{7}$ The second hypothesis tested was that those reactions that are preventable (such as ordering the wrong medication or giving the wrong dose) occurred in a pattern that 
was fundamentally different from those that accounted for the nonpreventable reactions, as those preventable reactions were not the result of an emergent process of a complex adaptive system.

\section{METHODS}

To test the first hypothesis that ADRs were described by a power law equation, the meta-analysis by Lazarou et $\mathrm{al}^{8}$ was analyzed, in which prospective studies of ADR from 1966 to 1996 were examined. Only those studies of ADRs that occurred among patients already in the hospital were used, to afford a clear idea of how many patients had a normal reaction to the drug. To allow greater discrimination between the different categories of reaction, only data from those studies that classified reaction severity as no ADR, nonserious, serious, and fatal reaction were used for the analyses performed in this article. Eight reports met these criteria and were included for analysis.

The data presented in the Lazarou et $\mathrm{al}^{8}$ paper were given in terms of percentages. To obtain the incidence of each type of reaction, these percentages were multiplied by the study size to generate the incidence of each type of reaction in each of the reports studied. Once incidences were obtained, they were summed for each category to give the overall incidences of each type of reaction in these 8 studies.

Summed data from the 8 papers presented in Lazarou et $\mathrm{l}^{8}$ were plotted on a log-log graph. Log incidence was plotted on the $y$-axis as a function of log severity, which increases along the $x$-axis. A log-log graph was used to allow easier identification of power law distribution of data (which appear as straight lines on such a graph). A best-fit trend line was generated for the data set by selecting the type of equation that best described the data and solving for its parameters by minimizing the sum of squares between the observed data and those predicted by the equation, and an $R^{2}$ value was calculated. This equation was then used to compare the observed data with those predicted by the derived equation, and a Pearson's correlation coefficient was calculated.

Data from each of the studies cited by Lazarou et al ${ }^{8}$ were then plotted individually in a similar manner and fit to power law equations as described above. ${ }^{9-16}$ These were plotted together on the same graph to allow comparison between the characteristics of each study and the summed data.

To compare the characteristics of this process in inpatients and outpatients, the data presented by Gurwitz et al ${ }^{17}$ which prospectively analyzed a cohort of elderly outpatients for ADR, were examined. Here, the categories of severity used were no reaction, significant, serious, life threatening, and fatal. Otherwise, these data were plotted and fit to equations as above.

To test the second hypothesis that those ADRs that are preventable are fundamentally different from those that are not, the Gurwitz et al ${ }^{17}$ paper was again analyzed. Data in this report were subclassified as to whether or not they were preventable. Incidences of preventable and nonpreventable reactions were plotted on a log-log axis as above and were also fit to equations in the same manner.

\section{RESULTS}

\section{Reaction Severity Follows a Power Law Distribution}

Summed data $(\mathrm{n}=19682$ ) from the 8 papers presented in Lazarou et $\mathrm{al}^{8}$ meeting criteria for this study report were plotted (Figure 1a). A power law equation was fit to these data, and the slope derived was equal to -4.02 . The equation fit the observed data with an $R^{2}$ value of 0.95 and a Pearson's correlation coefficient of 0.995 $(P<.01)$.

\section{This Power Law Distribution Persists Regardless of the Sample Size or Nature of the Population Studied}

Data from each of the studies cited by Lazarou et $\mathrm{al}^{8}$ were plotted individually, fit to power law equations as described above, and plotted together on the same graph to allow comparison between the characteristics of each of the studies and the summed data (Figure $1 \mathrm{~b}$ and Table I). The sample sizes used ranged from 379 to 11526 and included both pediatric and adult patients from a variety of inpatient clinical settings. All data sets fit well with a power law equation. The $R^{2}$ values ranged from 0.85 to 0.9999 , with a mean of 0.95 and a standard deviation of 0.050 . Pearson's correlation coefficients of observed data to those predicted by the equations ranged from 0.982 to 1 , with a mean value of 0.997 , a standard deviation of 0.006 , and $P$ values of $<.001$ to .034 . As can be seen graphically, the slopes obtained from these equations were all very similar, with a mean of -4.03 and a standard deviation of 0.36 .

\section{This Power Law Distribution Persists for Outpatients and Inpatients}

The outpatient data from the Gurwitz et al ${ }^{17}$ paper were plotted as above (Figure 2a). Axes are the same as those 


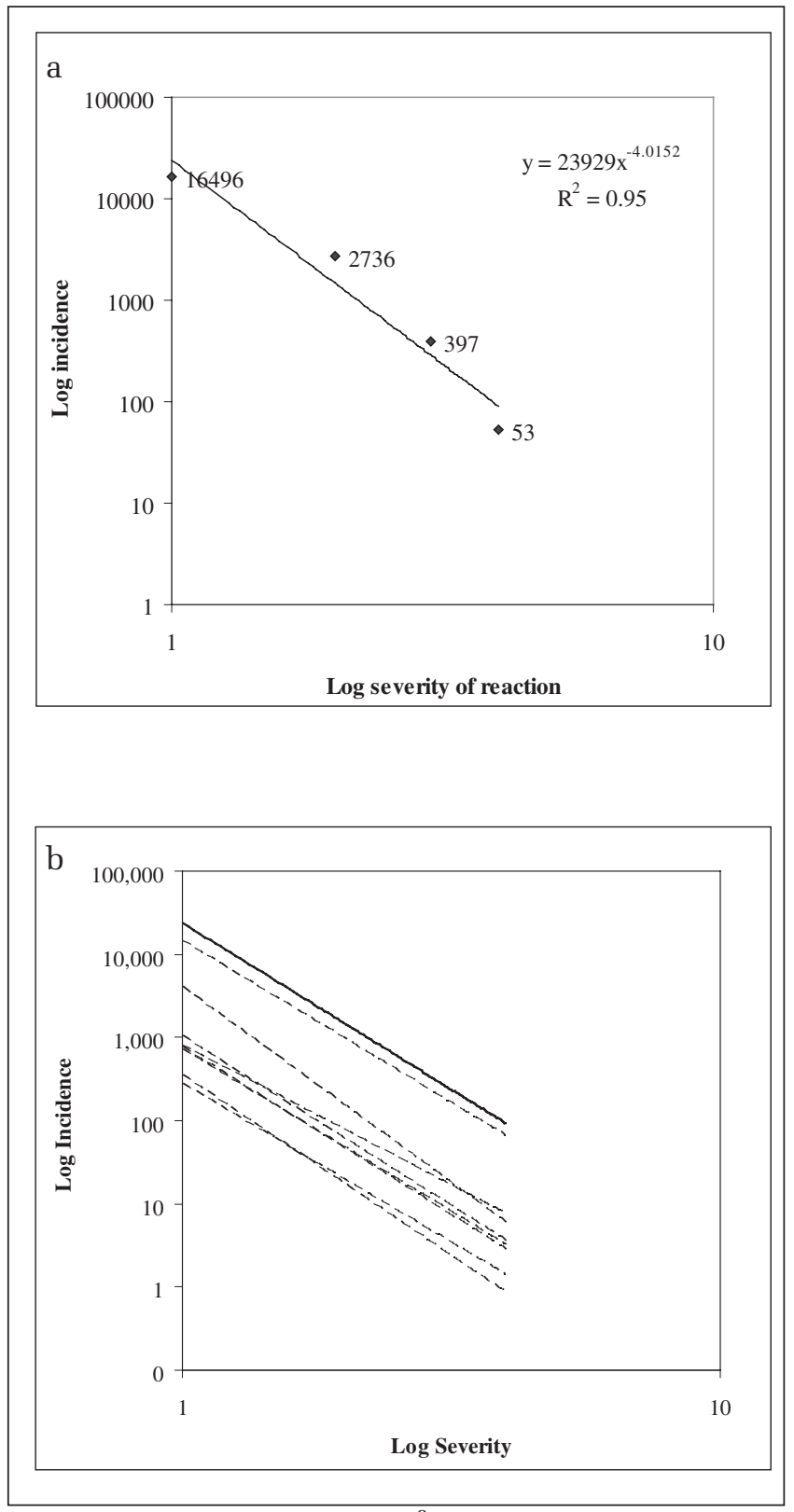

Figure 1. Data from Lazarou et al. ${ }^{8}$ (a) The sum of all reactions to medication categorized as follows. The incidence of a given reaction to medication is plotted as a function of its severity on a log-log axis ( $1=$ no reaction, $2=$ nonserious reaction, $3=$ serious reaction, and $4=$ fatal reaction). A trend line fit to these data is shown, along with its equation and fit. (b) The incidence of drug reaction was scale invariant and followed a similar power law distribution, regardless of sample size or the nature of the population studied. Summed data from the meta-analysis (bold) are plotted with the data from the individual studies (dashed).

used for the Lazarou et $\mathrm{al}^{8}$ data and those of the reports that contributed to it, with the exception that there were 5 categories of reaction in this analysis as com-
Table I The Slopes of Each Curve from the Individual Data Plotted in Figure 1

\begin{tabular}{|c|c|c|c|c|}
\hline Report & $\mathbf{n}$ & Slope & Pearson & $P$ \\
\hline Bates et $\mathrm{al}^{9}$ & 379 & -4.35 & 1 & $<.001$ \\
\hline Bates et $\mathrm{al}^{10}$ & 4031 & -4.70 & 1 & $<.001$ \\
\hline Bates et $\mathrm{al}^{11}$ & 420 & -3.82 & 0.999 & $<.034$ \\
\hline Miller $^{12}$ & 11526 & -3.90 & 0.982 & $<.018$ \\
\hline McKenzie et al ${ }^{13}$ & 658 & -3.91 & .999 & $<.001$ \\
\hline Gardner and Watson $^{14}$ & 939 & -3.36 & 1 & $<.001$ \\
\hline Seidl et al ${ }^{15}$ & 714 & -4.03 & 0.997 & $<.003$ \\
\hline Schimmel $^{16}$ & 1014 & -4.07 & 0.999 & $<.001$ \\
\hline Summed & 19682 & -4.02 & 0.995 & $<.005$ \\
\hline
\end{tabular}

pared with 4 in the former. The equation for the total reaction rate also fit well to a power law equation $\left(R^{2}=\right.$ 0.95 , Pearson's correlation coefficient of $1, P<.001$ ) and gave a slope of -4.04 , essentially identical to the slope of the summed reactions in Lazarou et al (which was -4.02).

\section{Preventable Adverse Drug Reactions Do Not Follow a Power Law Distribution}

A difference was noted when comparing the preventable with the nonpreventable reactions. The data on those reactions thought to be nonpreventable fit a power law equation well $\left(R^{2}=0.90\right.$, Pearson's correlation coefficient of $0.977, P=.023$ ) but with a greater negative slope of -5.04 . The fit of the preventable data to a power law equation was poor $\left(R^{2}=0.68\right.$, Pearson's correlation $=0.64, P=.36$ ), showing that preventable reactions do not follow a power law distribution. These data were then plotted on a nonlogarithmic graph and were found to fit well to a linear model, with an $R^{2}=$ 0.93, Pearson's correlation of 0.963 , and $P=.034$ (Figure 2b).

\section{DISCUSSION}

In physics, criticality is defined as a point at which the behavior of the system changes radically. This position has also been called "the edge of chaos."18 A good example of this is a phase transition between solid and liquid, where a small change in the system (in this example, the addition of heat) radically alters the state and behavior of the system: add a little heat to ice at $32^{\circ} \mathrm{F}$ and it changes to water; add the same amount of 


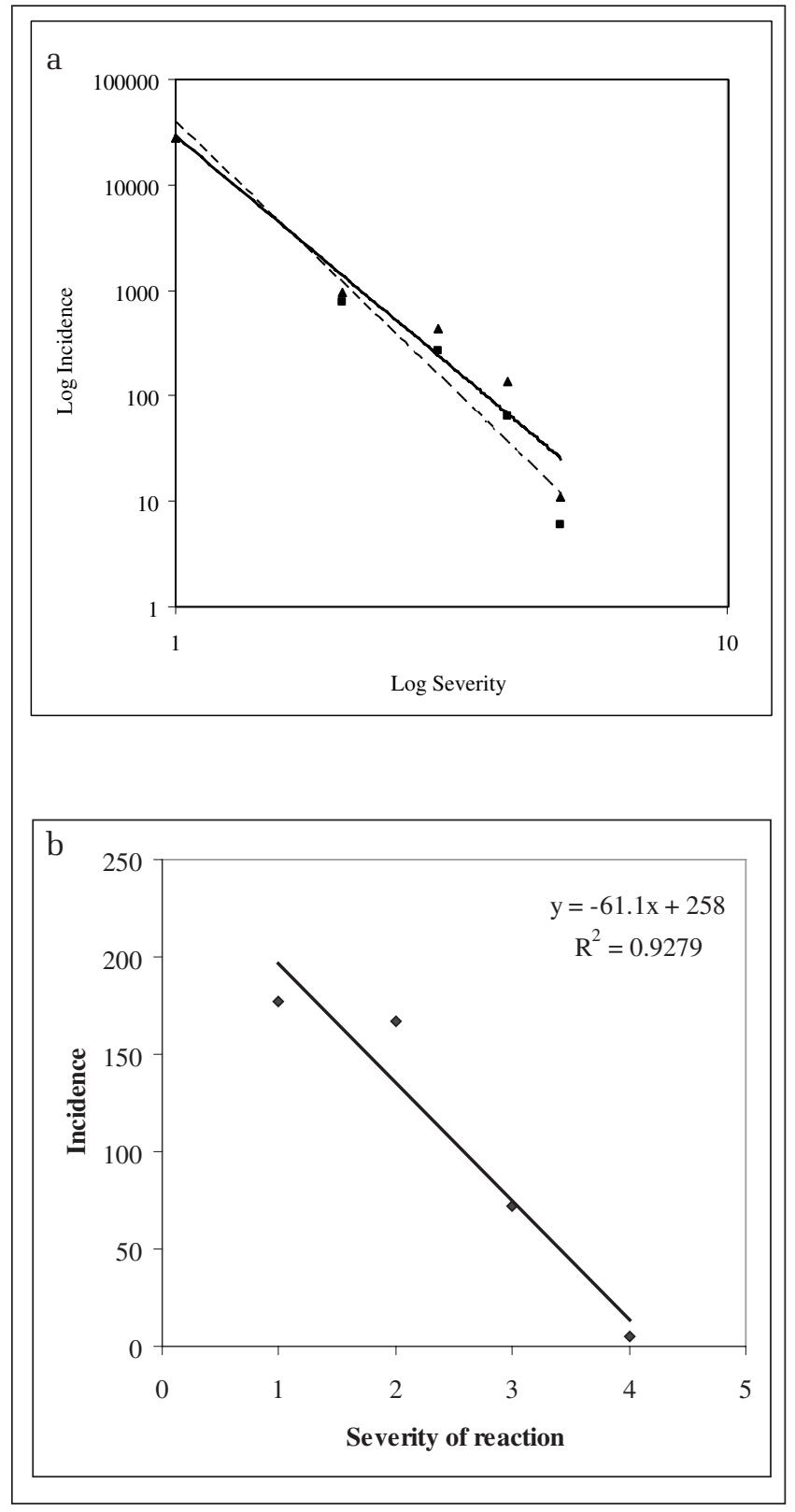

Figure 2. Data from Gurwitz et al. ${ }^{17}$ (a) Log severity of reaction is plotted against its log incidence as in Figure 1. The triangles and solid line represent the overall reaction rate, and the squares and dashed line represent nonpreventable reactions. The fit of the overall and nonpreventable reactions to the power law equation is good (Pearson's correlation of $1, P<.001$ and .997, $P=.023$, respectively). (b) Preventable reactions. Axes are no longer on a log scale. The preventable reactions fit a linear model very well on Cartesian axes (Pearson's of 0.963, $P=.034$ ).

heat to water at $33^{\circ} \mathrm{F}$ and all you get is water that is a little bit warmer. This location at the edge of chaos affords the system many advantages. Those systems that are too ordered will function well within a fairly limited range of environmental states or stimuli, but perturbations outside of this narrow confine will push the system beyond its adaptive ability to maintain homeostasis, and the system will fail. At the other extreme are those systems that are too chaotic to maintain the sort of dynamic nonequilibrium that is characteristic of living systems. Order provides memory, fluidity provides the ability to adapt to new situations, and adaptation to the edge of chaos allows the system as much flexibility as possible for handling new situations while still being able to pay the bills and maintain homeostasis.

The suspicion in conducting the research for this study was that we might have a similar sort of selforganized criticality that would be evidenced by examining the way in which people respond to the perturbation of taking a medication. Systems at the edge of criticality are identified by a power law distribution of their behavior when the incidence of the reaction to the perturbation in question is plotted as a function of its severity. ${ }^{7}$ This was the rationale for the investigations presented here. By showing that response to medication follows a power law distribution similar to other systems, it was hoped that this would provide some evidence to support the idea of self-organized criticality in pharmacotherapy and, in doing so, provide some explanation (albeit theoretical) for some of the variability we see in response to drugs.

The results presented here show that human responses to medication, ranging from salubrious to fatal, follow such a power law distribution, and therefore suggest that we have adapted to the edge of chaos in terms of our response to medications. There also appears to be some similarity in the distribution of these reactions, as all the data that included preventable and nonpreventable ADRs had very similar slopes, despite tremendous heterogeneity in the patient populations and the drugs used in them. This suggests that there is some common process at work in all the studied subjects.

This extrapolation of a linear relationship between categorical data (the rank of reaction severity) and continuous data (the incidence of reactions of that severity) may give the critical reader pause, but this approach has been successfully employed by many diverse disciplines. One of the best known of these is Zipf's law. George Zipf was a linguist who made the observation that for a given work of literature (in his initial report, he used James Joyce's Ulysses), the incidence of a given word was inversely proportional to its ranked frequency. ${ }^{20}$ When the words' incidences are plotted as a function of their ranked frequency on loglog axes, the familiar straight line of the power law ap- 
pears. Similar examples can be seen in the population of US cities, ${ }^{21}$ the number of visits to Web pages, ${ }^{22}$ and many others. ${ }^{7}$

So what does all this have to do with avalanches? The distribution of avalanches produced by a sand pile is a prime model of self-organized criticality. ${ }^{19} \mathrm{In}$ an experiment not too dissimilar to those we all performed as children, a pile of sand is allowed to form by dropping sand from a location centered above a table at a fixed rate. Over time, the pile will begin to increase in size until it arrives at a critical slope. The sand pile arrives at this critical slope independently, and the resultant criticality is therefore referred to as self-organized. At this point, addition of further sand to the system will cause avalanches to form. These avalanches vary in size and frequency, with a lot of smaller ones and a few very large ones. When the incidence of each avalanche is plotted as a function of its size on a log-log graph, a straight line emerges, and it becomes apparent that they are described by a power law equation as well, just as we have seen for total and nonpreventable ADRs. Both systems, human and sand pile, show evidence of adaptation to the edge of chaos.

Another interesting feature of these observations on the response to medication, and one that further supports the idea that they are evidence of some degree of self-organized criticality, is that they exhibit scale invariance. Scale invariance (also known as selfsimilarity) refers to the idea that the systems will appear similar at different scales: use a bigger table for the sand pile, and there will be some bigger avalanches, but there will still be a lot more small ones, and the slope of the pile and the distribution of the avalanches will be the same as for a smaller table. ${ }^{7}$ The sample sizes analyzed here ranged from 379 to 27617 , almost 2 orders of magnitude, but the distribution of response within each population and in the summed populations remained nearly identical, as evidenced by the very similar slopes of the equation's fit to each study's data. This scale invariance, in addition to the power law distribution of the reaction types, also supports the role of self-organized criticality in human response to medication.

A significant and interesting exception to this selforganized criticality is seen in the data provided by Gurwitz et al ${ }^{17}$ on medication reactions that were preventable. As seen in Figure 2b, those ADRs classified as preventable followed a linear model, not a power law one. This difference in distribution supports the idea that preventable medication errors are fundamentally different from those that are not.

This observation also suggests that preventable ADRs are just that: they can be prevented. The slope of a linear equation will eventually intercept the $x$-axis, meaning that the incidence of a given reaction will be zero. In contrast, those processes that are described by a power law never reach zero. They will approach it, but they always retain a nonzero value. Because ADRsspecifically, nonpreventable ADRs-seem to be described by such a law, this observation suggests that they ultimately cannot be prevented. They can probably be made less frequent, but their inevitability in some portion of our population taking medications will always remain. This susceptibility is a direct consequence of our adaptation to the edge of chaos.

But these observations are not all so pessimistic. Look at the data presented in Figure 2a and compare the slopes for the total reactions and those that were nonpreventable. By removing the preventable errors, the line describing the reactions becomes steeper and, in doing so, increases the percentage of milder reactions relative to the more severe ones. In the overall data presented by Gurwitz et al, ${ }^{17}$ there was 1 fatal reaction for every 86 significant ones (the mildest category defined). In the nonpreventable group, there was 1 fatal reaction for every 128 significant ones, whereas in the preventable group, the ratio was 1 to 35 . This supports the benefits to be gained by the ongoing efforts of physicians and institutions to decrease ADRs, as these preventable reactions seem to be disproportionately more severe than those that are not preventable.

This article has several potential limitations. One of the greatest of these is the nature of the data from which it draws. Although Lazarou et $\mathrm{al}^{8}$ have done a rigorous job of conducting their meta-analysis, there are still inherent problems in such a report. Some problems are introduced by having many investigators defining and classifying ADRs in very heterogeneous populations. The small number and subjective nature of the classifications of ADR used, employing such vague terms as mild or serious, introduces an element of imprecision and variability into both the definition of the ADRs and the assessment of their severity. Efforts to more precisely and quantitatively categorize ADR would be beneficial both to patient care and to future studies in this area.

Another factor that certainly figures into the observed pattern of ADR, and one that was not addressed in this study, is the regulatory process through which new drugs are introduced into and approved for human use. The progress of a drug through this process is largely informed by the characteristics of the biologic systems that it encounters during its evaluation. Thus, the regulatory process imposes certain restrictions on the frequencies and degrees of reaction seen in the drugs that reach the market and therefore may, to some 
degree, be a factor in the observed patterns of ADR. However, the magnitude of this factor relative to the effect of the characteristics of our adaptation is unclear and remains to be answered by future studies.

Many of the ideas suggested in this article are also largely speculative. Although the data presented here demonstrate the power law distribution of drug reactions and their scale invariance, they can only suggest that they are the result of self-organized criticality and cannot robustly prove it. Still, these sorts of observations gained from the perturbation of systems are the best and only methods we have at this time to identify those with self-organized criticality.

\section{CONCLUSION}

This article provides some explanation for the distribution of response to pharmacotherapy. This model, if correct, suggests a mechanism that explains the existence of ADRs. In doing so, it also explains the observation of many investigators and clinicians that milder drug reactions are more frequent than fatal ones. Last, it predicts that ADRs are an inevitable outcome in some percentage of the population who takes medications, while supporting ongoing efforts to reduce their frequency. More broadly, this model gives a glimpse into the nature of the organization that keeps us alive and gives us the ability to adapt to a wide array of new situations.

\section{REFERENCES}

1. Institute of Medicine. To Err Is Human: Building a Safer Health system. Washington, DC: National Academy Press; 1999.

2. Bates DW. Drugs and adverse drug reactions: how worried should we be? JAMA. 1998;279:1216-1217.

3. Bates DW. Medication errors: how common are they and what can be done to prevent them? Drug Saf. 1996;15:303-310.

4. Brennan TA, Leape LL, Laird NM, et al. Incidence of adverse events and negligence in hospitalized patients: results of the Harvard Medical Practice Study I. N Engl J Med. 1991;324:370-376.

5. Leape LL, Brennan TA, Laird N, et al. The nature of adverse events in hospitalized patients: results of the Harvard Medical Practice Study II. N Engl J Med. 1991;324:377-384.
6. Waldrop MM. Complexity: The Emerging Science at the Edge of Order and Chaos. New York: Simon \& Schuster; 1992.

7. Bak P. How Nature Works: The Science of Self-Organized Criticality. New York: Copernicus; 1996.

8. Lazarou J, Pomeranz BH, Corey PN. Incidence of adverse drug reactions in hospitalized patients: a meta-analysis of prospective studies. JAMA. 1998;279:1200-1205.

9. Bates DW, Cullen DJ, Laird N, et al. Incidence of adverse drug events and potential adverse drug events: implications for prevention. ADE Prevention Study Group. JAMA. 1995;274:29-34.

10. Bates DW, Boyle DL, Vander Vliet MB, Schneider J, Leape L. Relationship between medication errors and adverse drug events. J Gen Intern Med. 1995;10:199-205.

11. Bates DW, Leape LL, Petrycki S. Incidence and preventability of adverse drug events in hospitalized adults. J Gen Intern Med. 1993;8:289-294.

12. Miller RR. Drug surveillance utilizing epidemiologic methods: a report from the Boston Collaborative Drug Surveillance Program. Am J Hosp Pharm. 1973;30:584-592.

13. McKenzie MW, Stewart RB, Weiss CF, Cluff LE. A pharmacistbased study of the epidemiology of adverse drug reactions in pediatric medicine patients. Am J Hosp Pharm. 1973;30:898-903.

14. Gardner P, Watson LJ. Adverse drug reactions: a pharmacist-based monitoring system. Clin Pharmacol Ther. 1970;11:802-807.

15. Seidl LG, Thornton GF, Smith JW, Cluff LE. Studies on the epidemiology of adverse drug reactions, III: reactions in patients on a general medical service. Johns Hopkins Hosp Bull. 1966;119:299-315.

16. Schimmel EM. The hazards of hospitalization. Ann Intern Med. 1964;60:100-110.

17. Gurwitz JH, Field TS, Harrold LR, et al. Incidence and preventability of adverse drug events among older persons in the ambulatory setting. JAMA. 2003;289:1107-1116.

18. Kauffman SA. At Home in the Universe: The Search for Laws of Self-Organization and Complexity. New York: Oxford University Press; 1995.

19. Bak P, Tang C, Wiesenfeld K. Self-organized criticality: an explanation of the 1/f noise. Phys Rev Lett. 1987;59:381-384.

20. Zipf GK. Selected Studies of the Principle of Relative Frequency in Language. Cambridge, Mass: Harvard University Press; 1932.

21. US Census Bureau. Ranking tables for metropolitan areas, Table 3 : metropolitan areas ranked by population, 2000. Available at: www.census.gov/population/cen2000/phc-t3/tab03.pdf

22. Adamic LA, Huberman BA. The nature of markets in the World Wide Web. Q J Electron Comm. 2000;1:5-12. 